\title{
Existence of the Unique Nontrivial Solution for Mixed Fractional Differential Equations
}

\author{
Yujing Liu $(\mathbb{D}$, Chenguang Yan $(\mathbb{D}$, and Weihua Jiang $(\mathbb{D}$ \\ School of Sciences, Hebei University of Science and Technology, Shijiazhuang, Hebei 050018, China \\ Correspondence should be addressed to Chenguang Yan; ycgvbst@163.com
}

Received 1 March 2021; Revised 26 March 2021; Accepted 29 March 2021; Published 21 April 2021

Academic Editor: Maria Alessandra Ragusa

Copyright (c) 2021 Yujing Liu et al. This is an open access article distributed under the Creative Commons Attribution License, which permits unrestricted use, distribution, and reproduction in any medium, provided the original work is properly cited.

\begin{abstract}
In this paper, we consider the differential equations with right-sided Caputo and left-sided Riemann-Liouville fractional derivatives. Furthermore, the expression of Green's function is derived, and its properties are investigated. By the fixed-point theorem for both $\varphi-(h, e)$-concave operators and mixed monotone operators, we get the existence and uniqueness of the solution, respectively. As applications, some examples are provided to illustrate our main results.
\end{abstract}

\section{Introduction}

Fractional differential equations are generalization of the ordinary differential equations to a nonintegral order, and they have been widely used in other fields of mathematics (as discussed in [1-10]). In recent decades, many authors devoted themselves to fractional equations. More fractional boundary value problems have been applied to physics, biology, medicine, software engineering, neural network, and other related sciences (see [11-16]). With the publication of works discussed in [17-22], the theory of fractional boundary value problems are gradually enriched and systematized.

In [23], Song and Cui concerned the existence of solutions of nonlinear mixed fractional differential equation with the integral boundary value problem under resonance:

$$
\left\{\begin{array}{l}
{ }^{C} D_{1}^{\alpha} D_{0+}^{\beta} u(t)=f\left(t, u(t), D_{0+}^{\beta+1} u(t), D_{0+}^{\beta} u(t)\right), \quad 0<t<1, \\
u(0)=u^{\prime}(0)=0, \quad u(1)=\int_{0}^{1} u(t) d A(t),
\end{array}\right.
$$

where ${ }^{C} D_{1-}^{\alpha}$ is the left Caputo fractional derivative of order $\alpha \in(1,2]$, and $D_{0+}^{\beta}$ is the right Riemann-Liouville fractional derivate of order $\beta \in(0,1]$. The coincidence degree theory is the main theoretical basis to prove the existence of solutions of such problems. In recent years, there have been some studies on the existence of solutions for mixed fractional differential equations (see $[24,25]$ ).

By using the fixed-point theorem for mixed monotone operators, Jong et al. [26] dealt with the existence of positive solutions of the following multipoint boundary value problems for nonlinear fractional differential equations

$$
\left\{\begin{array}{l}
D_{0+}^{\beta} \varphi_{p}\left(D_{0+}^{\alpha} x(t)\right)=f(t, x(t)), \quad 0<t<1, \\
x(0)=0, D_{0+}^{\gamma} x(1)=\sum_{i=1}^{m-2} \xi i D_{0+}^{\gamma} x(\eta i), \\
D_{0+}^{\alpha} x(0)=0, \varphi_{p}\left(D_{0+}^{\alpha} x(1)\right)=\sum_{i=1}^{m-2} \zeta i \varphi_{p}\left(D_{0+}^{\alpha} x(\eta i)\right),
\end{array}\right.
$$

where $D_{0+}^{\alpha}, D_{0+}^{\beta}$, and $D_{0+}^{\gamma}$ are the standard Riemann-Liouville derivatives with $1<\alpha, \beta \leq 2,0<\gamma \leq 1,0<\xi i, \eta i, \zeta i<1, i=1$, $2, \cdots m-2$. The fixed-point theorem for mixed monotone operators was also used to prove the existence of solutions of boundary value problems $[27,28]$.

Moreover, many researchers focused on the $\varphi-(h, e)$ concave operators in [29-31] and its applications in [32]. 
Based on above works, this paper investigates the existence of solutions for the fractional differential equations

$$
\left\{\begin{array}{l}
{ }^{C} D_{1-}^{\alpha} D_{0+}^{\beta} x(t)+f(t, x(t))=b, \quad 0<t<1, \\
x(0)=0, x^{\prime}(1)=D_{0+}^{\beta} x(1)=0,
\end{array}\right.
$$

where ${ }^{C} D_{1-}^{\alpha}$ is the right-sided Caputo fractional derivative with $0<\alpha \leq 1, D_{0+}^{\beta}$ is the left-sided Riemann-Liouville fractional derivative with $1<\beta \leq 2$ and $\alpha+\beta>2$. Here, $f$ : $[0,1] \times(-\infty,+\infty) \longrightarrow(-\infty,+\infty)$ is continuous, and $b>0$ is a constant real number.

In Section 2, there are some definitions, properties, and lemmas related to this article. Then, we obtain the Green's function and prove some lemmas of Green's function. In Section 3, two important theorems are obtained. In Theorem 13 , set $P_{h, e}$ is defined. According to the fixed-point theorem of increasing concave operator, the existence of the unique solution of boundary value problem (3) is obtained. In Theorem 14, set $P_{h}$ is defined, the existence of solutions is also obtained by the fixed-point theorem of mixed monotone operators. In the last section, some examples are given to illustrate the validity of the theorems.

\section{Preliminaries}

In this part, we present some basic definitions, properties, and lemmas.

Definition 1 (see [20]). The left-sided and right-sided Riemann-Liouville fractional integrals of order $\alpha(\alpha>0)$ of a function $g:(0, \infty) \longrightarrow R$ are given by

$$
\begin{aligned}
& I_{0+}^{\alpha} \mathrm{g}(t)=\int_{0}^{t} \frac{(t-s)^{\alpha-1}}{\Gamma(\alpha)} \mathrm{g}(s) d s \\
& I_{1-\mathrm{g}}^{\alpha} \mathrm{g}(t)=\int_{t}^{1} \frac{(s-t)^{\alpha-1}}{\Gamma(\alpha)} \mathrm{g}(s) d s
\end{aligned}
$$

where $\Gamma(\alpha)$ is the Gamma function.
Definition 2 (see [20]). The left-sided Riemann-Liouville fractional derivative and right-sided Caputo fractional derivative of order $\alpha(\alpha>0)$ of a function $g \in C^{n}((0, \infty), R)$ are given by

$$
\begin{gathered}
D_{0+}^{\alpha} \mathrm{g}(t)=\frac{d^{n}}{d t^{n}}\left(I_{0+}^{n-\alpha} \mathrm{g}\right)(t), \\
{ }^{C} D_{1-\mathrm{g}}^{\alpha} \mathrm{g}(t)=(-1)^{n} I_{1-}^{n-\alpha} \mathrm{g}^{(n)}(t),
\end{gathered}
$$

where $n-1<\alpha<n$.

Property 3 (see [20]). Let $\alpha>0$ and $n=[\alpha]+1$. If $f(t) \epsilon$ $C^{n}[0,1]$, then

$$
\begin{gathered}
\left(I_{0+}^{\alpha}\right)\left(D_{0+}^{\alpha} f\right)(t)=f(t)+\sum_{j=1}^{n} C_{j} t^{\alpha-j}, \\
\left(I_{1-}^{\alpha}\right)\left({ }^{C} D_{1-}^{\alpha} f\right)(t)=f(t)+\sum_{k=0}^{n-1} C_{k}^{\prime}(1-t)^{k},
\end{gathered}
$$

where $C j, C_{k}^{\prime} \in R$ is arbitrary constant.

Lemma 4. Let $\alpha \in(0,1], \beta \in(1,2]$. For $y \in C[0,1]$, then the unique solution of the fractional differential equation

$$
\left\{\begin{array}{l}
{ }^{C} D_{1-}^{\alpha} D_{0+}^{\beta} x(t)=-y(t), \quad 0<t<1, \\
x(0)=0, x^{\prime}(1)=D_{0+}^{\beta} x(1)=0,
\end{array}\right.
$$

is $x(t)=\int_{0}^{1} G(t, s) y(s) d s$ where

$$
G(t, s)= \begin{cases}\frac{1}{\Gamma(\alpha) \Gamma(\beta)}\left[-\int_{0}^{s}(t-\tau)^{\beta-1}(s-\tau)^{\alpha-1} d \tau+t^{\beta-1} \int_{0}^{s}(1-\tau)^{\beta-2}(s-\tau)^{\alpha-1} d \tau\right], & 0 \leq s \leq t \leq 1 \\ \frac{1}{\Gamma(\alpha) \Gamma(\beta)}\left[-\int_{0}^{t}(t-\tau)^{\beta-1}(s-\tau)^{\alpha-1} d \tau+t^{\beta-1} \int_{0}^{s}(1-\tau)^{\beta-2}(s-\tau)^{\alpha-1} d \tau\right], & 0 \leq t \leq s \leq 1\end{cases}
$$

Proof. Applying the right-sided fractional integral $I_{1-}^{\alpha}$ to both sides of the Equation (7) and by Property 3, we can obtain that where $C_{0} \in R$ is arbitrary constant. Applying the left-sided fractional integral $I_{0+}^{\beta}$ to both sides Equation (9) above and by Property 3, we can obtain that

$$
x(t)=-I_{0+}^{\beta} I_{1-}^{\alpha} y(t)+\frac{C_{0}}{\Gamma(1+\beta)} t^{\beta}+C_{1} t^{\beta-1}+C_{2} t^{\beta-2},
$$


where $C i \in R(i=0,1,2)$. By $x(0)=0$ and $D_{0+}^{\beta} x(1)=0$, we get $C_{2}=C_{0}=0$, then

$$
x(t)=-I_{0+}^{\beta} I_{1-}^{\alpha} y(t)+C_{1} t^{\beta-1} .
$$

Finding the derivative of (11), we have

$$
x^{\prime}(t)=-I_{0+}^{\beta-1} I_{1-}^{\alpha} y(t)+(\beta-1) C_{1} t^{\beta-2},
$$

Since $x^{\prime}(1)=0$, it follows

$$
\begin{aligned}
x^{\prime}(1)= & \frac{-1}{\Gamma(\alpha) \Gamma(\beta-1)} \int_{0}^{1}(1-\tau)^{\beta-2} d \tau \int_{\tau}^{1}(s-\tau)^{\alpha-1} y(s) d s \\
& +(\beta-1) C_{1}=0 .
\end{aligned}
$$
have

Exchanging the order of the above double integral, we

$$
C_{1}=\frac{1}{\Gamma(\alpha) \Gamma(\beta)} \int_{0}^{1} y(s) d s \int_{0}^{s}(1-\tau)^{\beta-2}(s-\tau)^{\alpha-1} d \tau .
$$

Substitute $C_{1}$ into (11), we know that

$$
\begin{aligned}
x(t)= & -\frac{1}{\Gamma(\alpha) \Gamma(\beta)} \int_{0}^{t}(t-\tau)^{\beta-1} d \tau \int_{\tau}^{1}(s-\tau)^{\alpha-1} y(s) d s \\
& +\frac{t^{\beta-1}}{\Gamma(\alpha) \Gamma(\beta)} \int_{0}^{1} y(s) d s \int_{0}^{s}(1-\tau)^{\beta-2}(s-\tau)^{\alpha-1} d \tau .
\end{aligned}
$$

Exchange the order of the first double integral in the above formula, we get that

$$
\begin{aligned}
x(t)= & \frac{-1}{\Gamma(\alpha) \Gamma(\beta)}\left[\int_{0}^{t} y(s) d s \int_{0}^{s}(t-\tau)^{\beta-1}(s-\tau)^{\alpha-1} d \tau\right. \\
& \left.+\int_{t}^{1} y(s) d s \int_{0}^{t}(t-\tau)^{\beta-1}(s-\tau)^{\alpha-1} d \tau\right] \\
& +\frac{t^{\beta-1}}{\Gamma(\alpha) \Gamma(\beta)} \int_{0}^{1} y(s) d s \int_{0}^{s}(1-\tau)^{\beta-2}(s-\tau)^{\alpha-1} d \tau .
\end{aligned}
$$

Calculated that the Green's function of the fractional differential Equation (7) is

$$
G(t, s)= \begin{cases}\frac{1}{\Gamma(\alpha) \Gamma(\beta)}\left[-\int_{0}^{s}(t-\tau)^{\beta-1}(s-\tau)^{\alpha-1} d \tau+t^{\beta-1} \int_{0}^{s}(1-\tau)^{\beta-2}(s-\tau)^{\alpha-1} d \tau\right], & 0 \leq s \leq t \leq 1, \\ \frac{1}{\Gamma(\alpha) \Gamma(\beta)}\left[-\int_{0}^{t}(t-\tau)^{\beta-1}(s-\tau)^{\alpha-1} d \tau+t^{\beta-1} \int_{0}^{s}(1-\tau)^{\beta-2}(s-\tau)^{\alpha-1} d \tau\right], & 0 \leq t \leq s \leq 1\end{cases}
$$

The proof is completed.

Consequently, the boundary value problem (3) has a unique solution if and only if $x(t)$ satisfies the integral equation:

$$
\begin{aligned}
x(t) & =\int_{0}^{1} G(t, s)[f(s, x(s))-b] d s \\
& =\int_{0}^{1} G(t, s) f(s, x(s)) d s-b \int_{0}^{1} G(t, s) d s .
\end{aligned}
$$

Lemma 5. The Green's functions $G(t, s)$ defined by Lemma 4 satisfy the following properties:

(1) $G(t, s) \geq 0$, for all $s, t \in[0,1]$

(2) $(s / 2) J(s) t^{\beta-1} \leq G(t, s) \leq 2 J(s) t^{\beta-1} \leq J_{0} t^{\beta-1}$, for all $s, t$, $\in[0,1]$, where $J(s)=(1 /(\Gamma(\alpha) \Gamma(\beta))) \int_{s / 2}^{s}(1-\tau)^{\beta-2}$ $(s-\tau)^{\alpha-1} d \tau, J_{0}=2 /(\Gamma(\alpha) \Gamma(\beta)(\alpha+\beta-2))$.

Proof. First, we prove that the function $G(t, s)$ is nonnegative.
For any $0 \leq s \leq t \leq 1$,

$$
\begin{aligned}
G(t, s) & =\frac{1}{\Gamma(\alpha) \Gamma(\beta)} \int_{0}^{s}\left[t^{\beta-1}(1-\tau)^{\beta-2}-(t-\tau)^{\beta-1}\right](s-\tau)^{\alpha-1} d \tau \\
& \geq \frac{1}{\Gamma(\alpha) \Gamma(\beta)} \int_{0}^{s}\left[(t-t \tau)^{\beta-1}-(t-\tau)^{\beta-1}\right](s-\tau)^{\alpha-1} d \tau \geq 0 .
\end{aligned}
$$

For any $0 \leq t \leq s \leq 1$,

$$
\begin{aligned}
G(t, s)= & \frac{1}{\Gamma(\alpha) \Gamma(\beta)}\left[-\int_{0}^{t}(t-\tau)^{\beta-1}(s-\tau)^{\alpha-1} d \tau\right. \\
& \left.+t^{\beta-1} \int_{0}^{s}(1-\tau)^{\beta-2}(s-\tau)^{\alpha-1} d \tau\right] \\
\geq & \frac{1}{\Gamma(\alpha) \Gamma(\beta)}\left[-\int_{0}^{s}(t-\tau)^{\beta-1}(s-\tau)^{\alpha-1} d \tau\right. \\
& \left.+t^{\beta-1} \int_{0}^{s}(1-\tau)^{\beta-2}(s-\tau)^{\alpha-1} d \tau\right] \geq 0 .
\end{aligned}
$$

In a word, for any $t, s \in[0,1], G(t, s) \geq 0$.

Then, we prove $G(t, s) \leq 2 J(s) t^{\beta-1} \leq J_{0} t^{\beta^{-1}}$. 
For any $t, s \in[0,1]$,

$$
\begin{aligned}
G(t, s) \leq & \frac{t^{\beta-1}}{\Gamma(\alpha) \Gamma(\beta)} \int_{0}^{s}(1-\tau)^{\beta-2}(s-\tau)^{\alpha-1} d \tau \\
= & \frac{t^{\beta-1}}{\Gamma(\alpha) \Gamma(\beta)}\left[\int_{0}^{\frac{s}{2}}(1-\tau)^{\beta-2}(s-\tau)^{\alpha-1} d \tau\right. \\
& \left.+\int_{\frac{s}{2}}^{s}(1-\tau)^{\beta-2}(s-\tau)^{\alpha-1} d \tau\right] \\
\leq & \frac{2 t^{\beta-1}}{\Gamma(\alpha) \Gamma(\beta)} \int_{\frac{s}{2}}^{s}(1-\tau)^{\beta-2}(s-\tau)^{\alpha-1} d \tau \\
= & 2 J(s) t^{\beta-1} \leq \frac{2 t^{\beta-1}}{\Gamma(\alpha) \Gamma(\beta)} \int_{\frac{s}{2}}^{s}(s-\tau)^{\alpha+\beta-3} d \tau \\
= & \frac{2 t^{\beta-1} s^{\alpha+\beta-2}}{\Gamma(\alpha) \Gamma(\beta)(\alpha+\beta-2) 2^{\alpha+\beta-2}} \leq J_{0} t^{\beta-1} .
\end{aligned}
$$

Finally, we prove that $G(t, s) \geq(s / 2) J(s) t^{\beta-1}$. For any $t, s \in[0,1]$,

$$
\begin{aligned}
G(t, s) \geq & \frac{1}{\Gamma(\alpha) \Gamma(\beta)}\left[-\int_{0}^{s}(t-\tau)^{\beta-1}(s-\tau)^{\alpha-1} d \tau\right. \\
& \left.+t^{\beta-1} \int_{0}^{s}(1-\tau)^{\beta-2}(s-\tau)^{\alpha-1} d \tau\right] \\
= & \frac{t^{\beta-1}}{\Gamma(\alpha) \Gamma(\beta)} \int_{0}^{s}\left[(1-\tau)^{\beta-2}-\left(1-\frac{\tau}{t}\right)^{\beta-1}\right](s-\tau)^{\alpha-1} d \tau \\
\geq & \frac{\int_{0}^{s}\left[(1-\tau)^{\beta-2}-(1-\tau)^{\beta-1}\right](s-\tau)^{\alpha-1} d \tau}{\Gamma(\alpha) \Gamma(\beta)} t^{\beta-1} \\
= & \frac{\int_{0}^{s} \tau(1-\tau)^{\beta-2}(s-\tau)^{\alpha-1} d \tau}{\Gamma(\alpha) \Gamma(\beta)} t^{\beta-1} \\
\geq & \frac{\int_{s / 2}^{s}(1-\tau)^{\beta-2}(s-\tau)^{\alpha-1} d \tau}{\Gamma(\alpha) \Gamma(\beta)} t^{\beta-1} \\
\geq & \frac{s}{2} \frac{\int_{s / 2}^{s}(1-\tau)^{\beta-2}(s-\tau)^{\alpha-1} d \tau}{\Gamma(\alpha) \Gamma(\beta)} t^{\beta-1} \\
= & \frac{s}{2} J(s) t^{\beta-1} .
\end{aligned}
$$

The proof is completed.

Next, we summarize two fixed-point lemmas and some basic concepts in ordered Banach space.

Let $(E,\|\cdot\|)$ be a real Banach space which is partially ordered by a cone $P \subset E$, i.e., $x \leq y$ if and only if $y-x \in P$. If $x \leq y$ and $x \neq y$, then we denote $x<y$ or $y>x$. $\theta$ denotes the zero element of $E$. $P$ is called normal if there exists $M>0$ such that, for all $x, y \in E, \theta \leq x \leq y$ implies $\|x\| \leq$ $M\|y\|$; in this case, $M$ is called the normality constant of $P$. We say that an operator $A: E \longrightarrow E$ is increasing if $x \geq y$ implies $A x \geq A y$ [29].
Given $h \in E$ and $h>\theta$, we define the set

$$
P_{h}=\left\{x \in E \mid \exists 0<\lambda_{1} \leq \lambda_{2}: \lambda_{1} h \leq x \leq \lambda_{2} h\right\} .
$$

Remark 6. If $x \in P_{h}$, let $\lambda_{0}=\max \left\{1 / \lambda_{1}, \lambda_{2}\right\}$, we have $\left(1 / \lambda_{0}\right)$ $h \leq x \leq \lambda_{0} h$.

Let $e \in P$ and $\theta \leq e \leq h$, we define the set

$$
P_{h, e}=\left\{x \in E \mid \exists 0<\mu_{1} \leq \mu_{2}: \mu_{1} h \leq x+e \leq \mu_{2} h\right\} .
$$

Definition 7 (see $[29,31]$ ). Let $A: P \longrightarrow P$ be a given operator. For any $x \in P$ and $r \in(0,1)$, there exists $\varphi(r) \in(r, 1)$ such that $A(r x) \leq \varphi(r) A x$. Then, $A$ is called a generalized concave operator.

Definition 8 (see [29]). Let $A: P_{h, e} \longrightarrow E$ be a given operator. For any $x \in P_{h, e}$ and $\lambda \in(0,1)$, there exists $\varphi(\lambda)>\lambda$ such that

$$
A(\lambda x+(\lambda-1) e) \geq \varphi(\lambda) A x+(\varphi(\lambda)-1) e .
$$

Then, $A$ is called a $\varphi-(h, e)$-concave operator.

Lemma 9 (see [29]). Let $P$ be normal and $A$ be an increasing $\varphi-(h, e)$-concave operator with $A h \in P_{h, e}$. Moreover, for any $\omega_{0} \in P_{h, e}$, making the sequence $\omega_{n}=A \omega_{n-1}, n=1,2, \cdots$, then we obtain $\left\|\omega_{n}-x^{*}\right\| \longrightarrow 0$ as $n \longrightarrow \infty$.

Definition 10 (see [28]). Let $D \subset E$. Operator $A: D \times D \longrightarrow E$ is said to be mixed monotone if $A(x, y)$ is nondecreasing in $x$ and nonincreasing in $y$, i.e., $x_{1} \leq x_{2}, y_{2} \leq y_{1}, x_{i}, y_{i} \in D(i=1,2)$ implies $A\left(x_{1}, y_{1}\right) \leq A\left(x_{2}, y_{2}\right)$.

Definition 11 (see [28]). Let $A: P \times P \longrightarrow E$ a mixed monotone operator. Assume that for all $0<t<1$, there exists $0<\sigma=\sigma(t)<1$ such that

$$
A\left(t x, \frac{1}{t} y\right) \geq t^{\sigma(t)} A(x, y)
$$

holds for all $x, y \in P$; then, $A$ is called a $t-\sigma(t)$ mixed monotone model operator.

Lemma 12 (see [28]). Let $h>\theta . A: P_{h} \times P_{h} \longrightarrow P_{h}$ is a $t-\sigma(t)$ mixed monotone operator. Then, $A$ has exactly one fixed-point $x^{*}$ in $P_{h}$. Moreover, constructing successively the sequences

$$
x_{n}=A\left(x_{n-1}, y_{n-1}\right), y_{n}=A\left(y_{n-1}, x_{n-1}\right), \quad n=1,2, \cdots,
$$

for any initial point $x_{0}, y_{0} \in P_{h}$, we have $\left\|x_{n}-x^{*}\right\| \longrightarrow 0$ and $\left\|y_{n}-x^{*}\right\| \longrightarrow 0$ as $n \longrightarrow \infty$.

\section{Main Results}

We consider the space $E=C[0,1]$ with the usual maximum norm $\|u\|=\max _{t \in[0,1]}|u(t)|$. Clearly, $E$ is a Banach space. Set $P=\{x \in E \mid x(t) \geq 0, t \in[0,1]\}$. Obviously, $P$ is a normal cone and $P \subset E$, the normality constant is 1 . 
Theorem 13. Let $h$ and $e$ are defined by $h(t)=H t^{\beta-1}, e(t)=$ $b \int_{0}^{1} G(t, s) d s$, where $t \in[0,1]$ and $H=2 b /(\Gamma(\alpha) \Gamma(\beta)(\alpha+\beta-$ $2)), G(t, s)$ is the Green's functions of (3) defined by Lemma 4. Suppose the following conditions hold:

(H1). $f:[0,1] \times\left[-e^{*},+\infty\right] \longrightarrow(-\infty,+\infty)$ is continuous and increasing with respect to the second variable, that is, for any $0 \leq x \leq y$, we get $f(t, x(t)) \leq f(t, y(t))$, where $e^{*}=$ $\max _{0 \leq t \leq 1} e(x)$

(H2). For any $\lambda \in(0,1)$ there is $\varphi(\lambda)>\lambda$ such that $f(t, \lambda x+(\lambda-1) y) \geq \varphi(\lambda) f(t, x)$, where $t \in[0,1], x \in[0,+\infty)$, $y \in\left[0, e^{*}\right]$

(H3). $f(t, 0) \geq 0$ with $f(t, 0) \not \equiv$ for $t \in[0,1]$.

Then, the fractional differential equations (3) have a unique nontrivial solution $x^{*}$ in $P_{h, e}$. Moreover, for any given initial value $x_{0} \in P_{h, e}$, making the sequence $x_{n}=A x_{n-1}, n=$ $1,2, \cdots$, then we obtain $\left\|x_{n}-x^{*}\right\| \longrightarrow 0$ as $n \longrightarrow \infty$.

Proof. For any $\in[0,1]$, it is easy to see $e(t) \geq 0$, that is, $e \in P$. Further,

$$
e(t) \leq b \int_{0}^{1} J_{0} t^{\beta-1} d s=b \frac{2}{\Gamma(\alpha) \Gamma(\beta)(\alpha+\beta-2)} t^{\beta-1}=h(t) .
$$

Hence, $0 \leq e(t) \leq h(t)$.

Let

$$
A x(t)=\int_{0}^{1} G(t, s) f(s, x(s)) d s-e(t), \quad t \in[0,1] .
$$

The boundary (3) has an integral formulation given by

$$
\begin{aligned}
x(t) & =\int_{0}^{1} G(t, s) f(s, x(s)) d s-b \int_{0}^{1} G(t, s) d s \\
& =\int_{0}^{1} G(t, s) f(s, x(s)) d s-e(t)=A x(t) .
\end{aligned}
$$

So, $x(t)$ is the solution of the problem (3) if and only if $x(t)$ is the fixed point of the operator of $A$.

Firstly, it is apparent from the definition of $A$ that $A$ is $P_{h, e} \longrightarrow E$.

From (H2), we know that

$$
\begin{aligned}
A( & \lambda x+(\lambda-1) e) \\
& =\int_{0}^{1} G(t, s) f[s, \lambda x(s)+(\lambda-1) e(s)] d s-e(t) \\
& \geq \int_{0}^{1} G(t, s) \varphi(\lambda) f(s, x(s)) d s-e(t) \\
& =\varphi(\lambda) \int_{0}^{1} G(t, s) f(s, x(s)) d s-\varphi(\lambda) e(t)+\varphi(\lambda) e(t)-e(t) \\
& =\varphi(\lambda)\left[\int_{0}^{1} G(t, s) f(s, x(s)) d s-e(t)\right]+[\varphi(\lambda)-1] e(t) \\
& =\varphi(\lambda) A x(t)+[\varphi(\lambda)-1] e(t) .
\end{aligned}
$$

By Definition $8, A$ is $\varphi-(h, e)$-concave operator.
Secondly, for $x, y \in P_{h, e}$ and $x \leq y$, we get

$$
\begin{aligned}
A x(t) & =\int_{0}^{1} G(t, s) x(s) d s-e(t) \leq \int_{0}^{1} G(t, s) y(s) d s-e(t) \\
& =A y(t) .
\end{aligned}
$$

So, $A$ is increasing.

Thirdly, we would prove that $A h \in P_{h, e}$.

Let $l_{1}=\left(\int_{0}^{1} s / 2\left[(1-(s / 2))^{\alpha+\beta-2}-(1-s)^{\alpha+\beta-2}\right] f(s, 0) d s\right) /$ $(H \Gamma(\alpha) \Gamma(\beta)(\alpha+\beta-2)), l_{2}=\left(2 \int_{0}^{1} f(s, H) d s\right) /(H \Gamma(\alpha) \Gamma(\beta)(\alpha$ $+\beta-2))$.

$$
\begin{aligned}
A h(t)+e(t) & =\int_{0}^{1} G(t, s) f(s, h(s)) d s \\
& =\int_{0}^{1} G(t, s) f\left(s, H s^{\beta-1}\right) d s \\
& \leq \int_{0}^{1} J_{0} t^{\beta-1} f(s, H) d s \\
& \leq \frac{2}{H \Gamma(\alpha) \Gamma(\beta)(\alpha+\beta-2)} \int_{0}^{1} f(s, H) d s H t^{\beta-1} \\
& =l_{2} h(t), \\
A h(t)+e(t) & =\int_{0}^{1} G(t, s) f\left(s, H s^{\beta-1}\right) d s \\
& \geq \int_{0}^{1} \frac{s}{2} J(s) t^{\beta-1} f(s, 0) d s \\
& \geq \frac{\int_{0}^{1} s / 2\left[(1-(s / 2))^{\alpha+\beta-2}-(1-s)^{\alpha+\beta-2}\right] f(s, 0) d s}{H \Gamma(\alpha) \Gamma(\beta)(\alpha+\beta-2)} H t^{\beta-1} \\
& =l_{1} h(t) .
\end{aligned}
$$

It is not difficult to verify that

$$
\begin{aligned}
0 & <\frac{\int_{0}^{1} s / 2\left[(1-(s / 2))^{\alpha+\beta-2}-(1-s)^{\alpha+\beta-2}\right] f(s, 0) d s}{H \Gamma(\alpha) \Gamma(\beta)(\alpha+\beta-2)} \\
& \leq \frac{2 \int_{0}^{1} f(s, H) d s}{H \Gamma(\alpha) \Gamma(\beta)(\alpha+\beta-2)} .
\end{aligned}
$$

That is to say, $l_{2} \geq l_{1}>0$. Then, we have $l_{1} h \leq A h+e$ $\leq l_{2} h$. So, $A h \in P_{h, e}$.

Consequently, by using Lemma 9 , the operator $A$ has a unique fixed-point $x^{*}$ in $P_{h, e}$, i.e., $x^{*}(t)=\int_{0}^{1} G(t, s) f\left(s, x^{*}(s)\right)$ $d s-e(t), t \in[0,1]$. And for any $x_{0} \in P_{h, e}$, there exists the sequence $x_{n}=A x_{n-1}, n=1,2, \cdots$, satisfies $x_{n} \longrightarrow x^{*}$ as $n \longrightarrow \infty$,

$$
x_{n}=\int_{0}^{1} G(t, s) f\left(s, x_{n-1}(s)\right) d s-b \int_{0}^{1} G(t, s) d s .
$$

The proof is completed.

Theorem 14. Let $h(t)=H t^{\beta-1}$, where $t \in[0,1]$ and $H$ is defined by Theorem 13. Suppose the following conditions hold: 
(H4). $f(t, x)-b=\phi(t, x)+\psi(t, x) \geq 0$, where $\phi, \psi:[0,1]$ $\times(0,+\infty) \longrightarrow[0,+\infty)$ are continuous functions and for any fixed $t \in[0,1], \phi(t, x)$ is nondecreasing in $x>0$, and $\psi(t, x)$ is nonincreasing in $x>0$

(H5). For $0<\eta<1$, there exists $\sigma \in(0, \beta)$ such that $\phi(t$, $\eta x) \geq \eta^{\sigma} \phi(t, x), \psi\left(t, \eta^{-1} x\right) \geq \eta^{\sigma} \psi(t, x)$. For $\eta>1$, from above inequality, we can get that $\phi(t, \eta x) \leq \eta^{\sigma} \phi(t, x), \psi\left(t, \eta^{-1} x\right) \leq$ $\eta^{\sigma} \psi(t, x)$, where $t \in[0,1]$ and $x>0$;

(H6) $\int_{0}^{1} t^{-\sigma(\beta-1)} \psi(t, H) d t<+\infty$.

Then, the boundary value problem (3) has a unique positive solution in $P_{h}$.

Proof. According to definition of $P_{h}$ in Theorem 13, $P_{h}$ is normal cone. Let

$$
\begin{aligned}
T(x, y)(t) & =\int_{0}^{1} G(t, s)[f(s, x(s))-b] d s \\
& =\int_{0}^{1} G(t, s)[\phi(s, x(s))+\psi(s, y(s))] d s .
\end{aligned}
$$

From Remark 6, for any $x, y \in P_{h}$, there exist two positive constants $M_{1}$ and $M_{2}$ such that $\left(1 / M_{1}\right) h(t) \leq x(t) \leq M_{1} h(t)$, $\left(1 / M_{2}\right) h(t) \leq y(t) \leq M_{2} h(t)$. Let $M=\max \left\{M_{1}, M_{2}\right\}$, it is easy to know that $M>1$ and

$$
\frac{1}{M} h(t) \leq x(t) \leq M h(t), \frac{1}{M} h(t) \leq y(t) \leq M h(t) .
$$

By (H5), we have

$$
\begin{aligned}
\phi(t, x(t)) & \leq \phi(t, M h(t)) \leq \phi(t, M H) \leq M^{\sigma} \phi(t, H), \\
\phi(t, x(t)) & \geq \phi\left(t, \frac{1}{M} h(t)\right)=\phi\left(t, \frac{1}{M} H t^{\beta-1}\right) \\
& \geq M^{-\sigma} t^{\sigma(\beta-1)} \phi(t, H), \\
\psi(t, y(t)) & \leq \psi\left(t, \frac{1}{M} h(t)\right)=\psi\left(t, \frac{1}{M} H t^{\beta-1}\right) \\
& \leq M^{\sigma} t^{-\sigma(\beta-1)} \psi(t, H), \\
\psi(t, y(t)) & \geq \psi(t, M h(t)) \geq \psi(t, M H) \geq M^{-\sigma} \psi(t, H) .
\end{aligned}
$$

Hence, for any $t \in[0,1]$, we have the followings:

$$
\begin{aligned}
T(x, y)(t) & =\int_{0}^{1} G(t, s)[\phi(s, x(s))+\psi(s, y(s))] d s \\
& \leq \int_{0}^{1} J_{0} t^{\beta-1}\left[M^{\sigma} \phi(s, H)+M^{\sigma} s^{-\sigma(\beta-1)} \psi(s, H)\right] d s \\
& =J_{0} M^{\sigma} \int_{0}^{1}\left[\phi(s, H)+s^{-\sigma(\beta-1)} \psi(s, H)\right] d s t^{\beta-1} \\
& =H^{-1} J_{0} M^{\sigma} \int_{0}^{1}\left[\phi(s, H)+s^{-\sigma(\beta-1)} \psi(s, H)\right] d s h(t) .
\end{aligned}
$$

It follows from the above $(H 6)$ that $T(x, y)(t)<+\infty$.

$$
\begin{aligned}
T(x, y)(t) & \geq \int_{0}^{1} \frac{s}{2} J(s) t^{\beta-1}[\phi(s, x(s))+\psi(s, y(s))] d s \\
& \geq \int_{0}^{1} \frac{s}{2} J(s) t^{\beta-1}\left[M^{-\sigma} s^{\sigma(\beta-1)} \phi(s, H)+M^{-\sigma} \psi(s, H)\right] d s \\
& =M^{-\sigma} \int_{0}^{1} \frac{s}{2} J(s) t^{\beta-1}\left[s^{\sigma(\beta-1)} \phi(s, H)+\psi(s, H)\right] d s t^{\beta-1} \\
& =H^{-1} M^{-\sigma} \int_{0}^{1} \frac{s}{2} J(s) t^{\beta-1}\left[s^{\sigma(\beta-1)} \phi(s, H)+\psi(s, H)\right] d s h(t) .
\end{aligned}
$$

Suppose $\quad m_{1}^{-1}=H^{-1} \int_{0}^{1}(s / 2) J(s) t^{\beta-1}\left[s^{\sigma(\beta-1)} \phi(s, H)+\psi(s\right.$, $H)] d s$,

$$
m_{2}=H^{-1} J_{0} \int_{0}^{1}\left[\phi(s, H)+s^{-\sigma(\beta-1)} \psi(s, H)\right] d s \text {, }
$$

then

$$
\frac{1}{m_{1} M^{\sigma}} h(t) \leq T(x, y)(t) \leq m_{2} M^{\sigma} h(t) .
$$

So, $T: P_{h} \times P_{h} \longrightarrow P_{h}$.

By (H4), we obtain that for any $x_{1} \leq x_{2}\left(x_{1}, x_{2} \in P_{h}\right)$ and $y \in P_{h}$,

$$
\begin{aligned}
T\left(x_{1}, y\right)(t) & =\int_{0}^{1} G(t, s)\left[\phi\left(s, x_{1}(s)\right)+\psi(s, y(s))\right] d s \\
& \leq \int_{0}^{1} G(t, s)\left[\phi\left(s, x_{2}(s)\right)+\psi(s, y(s))\right] d s \\
& =T\left(x_{2}, y\right)(t) .
\end{aligned}
$$

For any $y_{1} \leq y_{2}\left(y_{1}, y_{2} \in P_{h}\right)$ and $x \in P_{h}$,

$$
\begin{aligned}
T\left(x, y_{1}\right)(t) & =\int_{0}^{1} G(t, s)\left[\phi(s, x(s))+\psi\left(s, y_{1}(s)\right)\right] d s \\
& \geq \int_{0}^{1} G(t, s)\left[\phi(s, x(s))+\psi\left(s, y_{2}(s)\right)\right] d s \\
& =T\left(x, y_{2}\right)(t) .
\end{aligned}
$$

Consequently, $T$ is a mixed monotone operator. For any $\eta \in(0,1)$ and $\sigma \in(0, \beta)$ in $(H 5)$,

$\phi(t, \eta x(t)) \geq \eta^{\sigma} \phi(t, x(t)), \psi\left(t, \eta^{-1} x(t)\right) \geq \eta^{\sigma} \psi(t, x(t))$. 
So, we get

$$
\begin{aligned}
T\left(\eta x, \eta^{-1} y\right)(t) & =\int_{0}^{1} G(t, s)\left[\phi(s, \eta x(s))+\psi\left(s, \eta^{-1} y(s)\right)\right] d s \\
& \geq \eta^{\sigma} \int_{0}^{1} G(t, s)[\phi(s, x(s))+\psi(s, y(s))] d s \\
& =\eta^{\sigma} T(x, y)(t) .
\end{aligned}
$$

From Definition 11, $T$ is a $t-\sigma(t)$ mixed monotone model operator. By Lemma 12, $T$ has exactly one fixedpoint $x^{*}$ in $P_{h}$. Constructing successively the sequences

$$
x_{n}=T\left(x_{n-1}, y_{n-1}\right), y_{n}=T\left(y_{n-1}, x_{n-1}\right), \quad n=1,2, \ldots
$$

For any initial point $x_{0}, y_{0} \in P_{h}$, we have $\left\|x_{n}-x^{*}\right\| \longrightarrow 0$ and $\left\|y_{n}-x^{*}\right\| \longrightarrow 0$ as $n \longrightarrow \infty$.

The proof is completed.

\section{Application}

Now, we give two concrete examples to illustrate our main theorems.

Example 1. Consider the mixed fractional differential equation

$$
\left\{\begin{array}{l}
{ }^{C} D_{1-}^{3 / 4} D_{0+}^{3 / 2} x(t)+\left[\frac{e(t)}{e^{*}} x+e(t)\right]^{1 / 3}=1, \quad 0<t<1, \\
x(0)=0, x^{\prime}(1)=D_{0+}^{3 / 2} x(1)=0 .
\end{array}\right.
$$

Here, $\alpha=3 / 4, \beta=3 / 2, b=1>0$; therefore, $\alpha+\beta=9 / 4>2$, $e(t)=1 \times \int_{0}^{1} G(t, s) d s=\int_{0}^{1} G(t, s) d s>0, e^{*}=\max _{0 \leq t \leq 1} e(x), \quad H=$ $2 b /(\Gamma(\alpha) \Gamma(\beta)(\alpha+\beta-2))=8 /(\Gamma(3 / 4) \Gamma(3 / 2))=7.3665$.

(1) It is obvious that $f(t, x)=\left[\left(e(t) / e^{*}\right) x+e(t)\right]^{1 / 3}>0$ is continuous and increasing with respect to $x$

(2) Taking $\varphi(\lambda)=\lambda^{1 / 3}>\lambda$ for any $\lambda \in(0,1), t \in[0,1]$, $x \in[0,+\infty)$ and $y \in\left[0, e^{*}\right]$, we have

$$
\begin{aligned}
f(t & , \lambda x+(\lambda-1) y) \\
& =\left\{\frac{e(t)}{e^{*}}[\lambda x+(\lambda-1) y]+e(t)\right\}^{1 / 3} \\
& =\lambda^{1 / 3}\left\{\frac{e(t)}{e^{*}}\left[x+\left(1-\frac{1}{\lambda}\right) y\right]+\frac{1}{\lambda} e(t)\right\}^{1 / 3} \\
& =\lambda^{1 / 3}\left\{\frac{e(t)}{e^{*}} x+\left(1-\frac{1}{\lambda}\right) \frac{e(t)}{e^{*}} y+\frac{1}{\lambda} e(t)\right\}^{1 / 3} \\
& \geq \lambda^{1 / 3}\left\{\frac{e(t)}{e^{*}} x+\left(1-\frac{1}{\lambda}\right) \frac{e(t)}{e^{*}} e^{*}+\frac{1}{\lambda} e(t)\right\}^{1 / 3} \\
& =\lambda^{1 / 3}\left(\frac{e(t)}{e^{*}} x+e(t)\right)^{1 / 3}=\varphi(\lambda) f(t, x) .
\end{aligned}
$$

(3) Obviously, $f(t, 0)=[e(t)]^{1 / 3} \geq 0$, and $f(t, 0) \not \equiv 0$, for all $t \in[0,1]$.

Therefore, it follows from Theorem 13 that the problem (48) has a unique nontrivial solution $x^{*} \in P_{h, e}$, where $e(t)=$ $\int_{0}^{1} G(t, s) d s$ and $h(t)=7.3665 t^{\beta-1}$.

Example 2. Consider the mixed fractional differential equation

$$
\left\{\begin{array}{l}
{ }^{C} D_{1-}^{2 / 3} D_{0+}^{3 / 2} x(t)+\left[t\left(x+\frac{1}{x}\right)+\frac{1}{2}\right]+\left[\sqrt{t}\left(\sqrt{x}+\frac{1}{\sqrt{x}}\right)+\frac{1}{2}\right]=1, \quad 0<t<1, \\
x(0)=0, \quad x^{\prime}(1)=D_{0+}^{3 / 2} x(1)=0 .
\end{array}\right.
$$

Here, $\alpha=2 / 3, \beta=3 / 2, b=1>0$, therefore, $\alpha+\beta=(13 /$ 6) $>2, H=2 b /(\Gamma(\alpha) \Gamma(\beta)(\alpha+\beta-2))=12 /(\Gamma(2 / 3) \Gamma(3 / 2))=$ 9.9995 .

(1) It is obvious that $f(t, x)-1=[t(x+(1 / x))]+[\sqrt{t}$ $(\sqrt{x}+(1 / \sqrt{x}))] \geq 0$ is continuous. Letting $\phi(t, x)=$ $t x+\sqrt{t x} \geq 0, \psi(t, x)=(t / x)+\sqrt{(t / x)} \geq 0$, it is easy to know that for any $t \in[0,1], \phi(t, x)$ is nondecreasing in $x>0$, and $\psi(t, x)$ is nonincreasing in $x>0$

(2) For $\eta \in(0,1)$, there exists $\sigma=1 \in(0,3 / 2)$ such that $\phi(t, \eta x)=t \eta x+\sqrt{t \eta x} \geq \eta(t x+\sqrt{t x})=\eta \phi(t, x), \quad \psi(t$, $\left.\eta^{-1} x\right)=\eta(t / x)+\sqrt{\eta(t / x)} \geq \eta((t / x)+\sqrt{(t / x)})=\eta \psi(t$, $x)$, where $x>0$ and $t \in[0,1]$
(3) Obviously, for $H=9.9995, \psi(t, H)=(t / 9.9995)+$ $\sqrt{t / 9.9995}, \int_{0}^{1} t^{-\sigma(\beta-1)} \psi(t, H) d t=\int_{0}^{1} t^{-(1 / 2)}((t / 9.9995)$ $+\sqrt{t / 9.9995}) d t \approx 0.3829<+\infty$.

Therefore, it follows from Theorem 14 that the problem (50) has a unique nontrivial solution $x^{*} \in P_{h}$, where $h(t)=$ $9.9995 t^{\beta-1}$.

\section{Conclusion}

In this paper, we discuss the boundary value problem of mixed fractional differential equation with Caputo fractional derivatives and Riemann-Liouville fractional derivatives. 
Firstly, integrating Equation (7) and applying the boundary value conditions, we construct the Green's function of the boundary value problem. Unfortunately, the expression of the Green's function is very complex. Fortunately, we still prove the fundamental property of the Green's function.

Secondly, by using the fixed-point theorems of increasing convex operators which defined in set $P_{h, e}$, we obtain the existence of solution of the mixed fractional differential Equation (3). In addition, by using the fixed-point theorems of monotone mixed operators which defined in set $P_{h}$, we also obtain the existence of solution of the mixed fractional differential Equation (3). The two theorems restrict different assumptions on the nonlinear term $f$, respectively, and state the existence of solutions for the boundary value problem (3). These two theorems are not contradictory, but two cases. That gives the existence of solution proved in this paper more sufficiency.

At last, the validity of main theorems in this paper is obtained from the examples.

\section{Data Availability}

No data were used to support this study.

\section{Conflicts of Interest}

The authors declare that they have no competing interests.

\section{Authors' Contributions}

Each of the authors contributed to each part of this work equally. All the authors read and approved the final version of the manuscript.

\section{References}

[1] A. Çetinkaya, "The incomplete second Appell hypergeometric functions," Applied Mathematics and Computation, vol. 219, no. 15, pp. 8332-8337, 2013.

[2] G. M. N'Guérékata, T. Diagana, and A. Pankov, "Abstract differential and difference equations," Advances in Difference Equations, vol. 2010, Article ID 857306, 2 pages, 2010.

[3] E. Ilhan and I. O. Kiymaz, "A generalization of truncated $\mathrm{M}$-fractional derivative and applications to fractional differential equations," Applied Mathematics and Nonlinear Sciences, vol. 5, no. 1, pp. 171-188, 2020.

[4] A. Asif, H. Aydi, M. Arshad, and Z. Ali, "A novel picture fuzzyBanach space with some new contractive conditions and their fixed point results," Journal of Function Spaces, vol. 2020, Article ID 6305856, 12 pages, 2020.

[5] M. A. Ragusa, "Commutators of fractional integral operators on vanishing-Morrey spaces," Journal of Global Optimization, vol. 40, no. 1-3, pp. 361-368, 2008.

[6] E. Guariglia, "Riemann zeta fractional derivative - functional equation and link with primes," Advances in Difference Equations, vol. 2019, no. 1, 2019.

[7] M. I. Abbas and M. A. Ragusa, "On the hybrid fractional differential equations with fractional proportional derivatives of a function with respect to a certain function," Symmetry, vol. 13 , no. 2 , p. $264,2021$.
[8] E. Guariglia, "Harmonic symmetry of the Riemann zeta fractional derivative," AIP Conference Proceedings, vol. 2046, article 020035, 2018.

[9] C. Li, X. Dao, and P. Guo, "Fractional derivatives in complex planes," Nonlinear Analysis, vol. 71, no. 5-6, pp. 1857-1869, 2009.

[10] E. Guariglia and S. Silvestrov, "A functional equation for the Riemann zeta fractional derivative," AIP Conference Proceedings, vol. 1798, 2017.

[11] Y. Zhang, C. Cattani, and X. J. Yang, "Local fractional homotopy perturbation method for solving non-homogeneous heat conduction equations in fractal domains," Entropy, vol. 2015, no. 17, pp. 6753-6764, 2015.

[12] W. Gao, H. M. Baskonus, and L. Shi, "New investigation of bats-hostsreservoir-people coronavirus model and application to 2019-nCoV system," Advances in Difference Equations, vol. 2020, Article ID 391, 2020.

[13] W. Gao, P. Veeresha, D. G. Prakasha, and H. M. Baskonus, "Novel dynamic structures of 2019-nCoV with nonlocal operator via powerful computational technique," Biology, vol. 9, no. 5, pp. 1-14, 2020.

[14] W. Gao, P. Veeresha, D. G. Prakasha, H. M. Baskonus, and G. Yel, "New approach for the model describing the deathly disease in pregnant women using Mittag-Leffler function," Chaos, Solitons \& Fractals, vol. 134, article 109696, 2020.

[15] J. Singh, D. Kumar, Z. Hammouch, and A. Atangana, "A fractional epidemiological model for computer viruses pertaining to a new fractional derivative," Applied Mathematics and Computation, vol. 316, pp. 504-515, 2018.

[16] J. Wang, Y. Wen, Y. Gou, Z. Ye, and H. Chen, "Fractionalorder gradient descent learning of BP neural networks with Caputo derivative," Neural Networks, vol. 89, pp. 19-30, 2017.

[17] K. S. Miller and B. Ross, An Introduction to the Fractional Calculus and Fractional Differential Equations, John Wiley, New York, 1993.

[18] S. G. Samko, A. A. Kilbas, and O. I. Marichev, Fractional Integral and Derivatives: Theory and Applications, Gordon and Breach Science Publishers, Switzerland, 1993.

[19] Podlubny, Fractional Differential Equations: An Introduction to Fractional Derivatives, Fractional Differential Equations, to Methods of Their Solution and Some of Their Applications, Academic Press, 1998.

[20] A. Kilbas, H. M. Srivastava, and J. J. Trujillo, Theory and Applications of Fractional Differential Equations, Elsevier B. V, 2006.

[21] Guo, Nonlinear Problems in Abstract Cones, Academic Press, 1988.

[22] M. A. Krasnosel'skii and P. P. Zabreiko, Geometrical Methods of Nonlinear Analysis, Springer-Verlag, 1984.

[23] S. Song and Y. Cui, "Existence of solutions for integral boundary value problems of mixed fractional differential equations under resonance," Boundary Value Problems, vol. 2020, Article ID 23, 2020.

[24] A. G. Lakoud, R. Khaldi, and A. Kılıçman, "Existence of solutions for a mixed fractional boundary value problem," Advances in Difference Equations, vol. 2017, Article ID 164, 2017.

[25] S. Ahmad, K. Ntouyas, and A. Alsaedi, "Fractional order differential systems involving right Caputo and left RiemannLiouville fractional derivatives with nonlocal coupled 
conditions," Boundary Value Problems, vol. 2019, Article ID 109, 2019.

[26] S. Jong, H. C. Choi, and Y. H. Ri, "Existence of positive solutions of a class of multi-point boundary value problems for _p_-Laplacian fractional differential equations with singular source terms," Communications in Nonlinear Science and Numerical Simulation, vol. 72, pp. 272-281, 2019.

[27] D. Guo, "Existence and uniqueness of positive fixed points for mixed monotone operators and applications," Applicable Analysis, vol. 46, no. 1-2, pp. 91-100, 1992.

[28] Y. Wu and Z. Liang, "Existence and uniqueness of fixed points for mixed monotone operators with applications," Nonlinear Analysis: Theory, Methods \& Applications, vol. 65, no. 10, pp. 1913-1924, 2006.

[29] Z. L. Wang, " $\phi-(h, e)$-concave operators and applications," Journal of Mathematical Analysis and Applications, vol. 454, no. 2, pp. 571-584, 2017.

[30] J. Ren and C. Zhai, "Some properties of sets, fixed point theorems in ordered product spaces and applications to a nonlinear system of fractional differential equations," Topological Methods in Nonlinear Analysis, vol. 49, no. 2, pp. 1-645, 2017.

[31] C. Zhai, C. Yang, and X. Zhang, "Positive solutions for nonlinear operator equations and several classes of applications," Mathematische Zeitschrift, vol. 266, no. 1, pp. 43-63, 2010.

[32] H. Wang and L. Zhang, "Uniqueness methods for the higherorder coupled fractional differential systems with multi-point boundary conditions," Bulletin des Sciences Mathmatiques, vol. 166, pp. 1-30, 2020. 